\title{
Role Of Intuition And Mindfulness On Problem - Solving:- A Review
}

\author{
Abirami Swaminathan, Dr. Maya Rathnasabapathy * \\ School of Social Sciences and Languages, Vellore Institute of Technology ( VIT ), Chennai- 600127.
}

Article History: Received:11 January 2021; Accepted: 27 February 2021; Published online: 5 April 2021

\begin{abstract}
The aim of this review was to understand the features and functions of two apparently conflicting constructs "intuition" and "mindfulness" with specific focus on problem solving. Literature published between 2010 and 2020 was searched and retrieved from data bases including Research Gate, PsycINFO, EBSCO, Crossref, Medline, PubMed and Scopus, using the key words "intuition", "mindfulness", "intuition and problem solving", "mindfulness and problem solving" and "intuition and mindfulness" for the study. The relationship between the two constructs was analysed within the dual process system of information processing. It was also investigated whether mindfulness would facilitate access to intuition and contribute to problem solving, or function complementary to each other. Analysis of published literature revealed that while the construct 'intuition' could be fitted into non-conscious system of cognitive process, the construct 'mindfulness' was related to conscious cognitive processing system, and therefore appeared to be contradictory. A critical evaluation of the relationship between the two constructs revealed a symbiotic relationship and emphasized the need to develop an integrated model to trigger intuition in mindful state with regard to problem-solving or decision-making.
\end{abstract}

Key words: Intuition, mindfulness, dual process theory, problem solving, decision making, integrated model, positive psychology.

\section{Introduction}

Martin Seligman (2002) introduced "Positive Psychology" with the goal of "scientific study of optimal human functioning [that] aims to discover and promote the factors that allow individuals and communities to thrive". To realize this goal, positive psychology focuses on scientific study on identifying the strengths of individuals and communities and providing with the tools and techniques to accomplish their goals and nurture their experiences and bring out the best in them to be successful in all walks of their life - be it personal, professional or social. What remains as an obstacle to realize the goal as the challenges / problems encountered in the everyday life. Therefore, the most fundamental requirement for well - being is effective problem - solving competency. Individuals must acquire the skills of problem-solving. The perception of "problem" and the strategies adopted by individual and communities to tackle them vary widely across domains. Several studies were reported on the psychological perspective of problem and problem-solving. A classification of problemsolving strategies mainly falls into two categories which are described within dual information processing cognitive system. The construct "intuition" facilitating non-conscious problem-solving process and the construct "mindfulness" which is related to awareness and conscious are analysed in this review.

\section{Method}

Many different computerized databases were searched which include Research Gate, PsycINFO, EBSCO, Crossref, PubMed, Medline, Scopus database and published research papers, dissertation and abstracts. Literature published between 2010 and 2020 on "mindfulness and problem-solving", "intuition and problemsolving", and "intuition and mindfulness" were analysed to find out the relationship between the two constructs with specific focus on problem-solving. Literature published before 2010 were used for conceptualizing and comparing the constructs.

\section{Problem and problem-solving}

The meaning / definition of the terms 'problem' and 'problem-solving' has different connotations in different situations, domains and age range. According to Allen Newell and Herbert Simon (1972), a more accommodative and inclusive definition of the term 'problem' "is any situation in which the state of affairs varies, or may in the future vary, from the desired state, and where there is no obvious way to reach the desired state". Newell and Simon (1972) defined "the conceptual area between the existing and desired states of affairs as a problem space and problem-solving is the cognitive process of navigating through the problem space through the virtual area between the actual or potential unsatisfactory state and the desired state". Though several different pathways might be available for navigation, they can be categorised into two main pathways 1 deliberate and 2 intuitive which are analysed in dual process theoretical framework. A survey of academic and professional literature revealed a variety of definitions including "something has gone wrong" (1981); "trying to move the world in the desired direction" (Lopez, 1984); to "situations where nothing has gone wrong yet, but where there is reason to believe that if some action is not taken and something may go wrong in the future". 
The theoretical frameworks describing the dual process information processing include "1. Cognitiveexperiential self-theory (Epstein, 1994, 2000); 2. Associative and Rule-based Processing (Bargh, 1989; Sloman, 1996; Smith \& DeCoster, 1999); 3. Automatic and controlled social cognition (Adolphs, 1999; Klein \& Kihlstrom, 1998; Ochsner \& Lieberman, 2001; Lieberman et al., 2004;); 4. System 1 and System 2 processes; (Stanovich and West) Schroyens et al., (2003); Stanovich, (1999); Stanovich \& West, (2000)".

According to Stanovich \& West (2000), the "intuitive pathway (System 1) processing is contextually dependent, associative, heuristic, tacit, intuitive and implicit/automatic in nature; hence, it is relatively undemanding in terms of its use of scarce cognitive resources". In contrast, "the deliberate pathway (System 2) processing is contextually independent, rule-based, analytic and explicit in nature; hence, it is relatively slow and makes great demands on cognitive resources". Both processes have their benefits and limitations.

An integrative approach would have a symbiotic effect in the process of problem-solving. 'Intuition' and 'mindfulness' are two constructs that have attracted the researchers for problem-solving and decisionmaking, working in diverse domains including executives in management, health care systems, education etc. An analysis of these constructs would reveal a contradictory nature between them but a critical evaluation would indicate that they can function symbiotically.

\section{Intuition - feature, functions and role in problem-solving}

Pretz \& Totz (2007) based on the analysis of theoretical and empirical studies on intuition which included both contemporary and historical studies, classified intuition as three distinct types: " 1 holistic, 2 inferential, and 3 affective". According to their classification all three types of intuitions are judgements but differ in the process associated with arriving at these judgements. Holistic judgements are arrived at involving qualitative, non-analytic process which integrates multiple and diverse inputs. The process associated with holistic intuitive judgement is not explicit in nature. Inferential intuition is related to the decision-making process which was analytic in nature but had become automated and intuitive. Affective intuition involves emotional reactions to problem situations.

Gerard P. Hodgkinson, Janice Langan-Fox and Eugene Sadler-Smith (2008) have critically analysed the relevance of intuition in behavioural sciences and distinguished intuition from related constructs such as insight, tacit knowledge, implicit learning and knowledge, instinct, insight and creativity and its place in dual processing system. They also identified the challenges in investigating intuition with regard to theoretical frameworks and methods for valid and reliable empirical research. The common features observed in various definitions of intuition as consolidated by Erik Dane Jesse H. Jones (2009) include "(1) non-conscious information processing, (2) holistic associations, (3) affect, and (4) speed".

Erik Dane and Jesse H. Jones (2009) based on the function, nature of association; affect and speed identified three types of intuition: 1. Problem-solving intuition, 2. Moral intuition, and 3. Creative intuition. Julie Gore and Eugene Sadler-Smith in addition to these three types of intuitions included social intuition based on process - outcome framework.

Viktor Dörfler and Fran Ackermann (2012) based on knowledge or emotions associated with the process further classified each of these intuitions into two as intuitive judgement and intuitive insight.

Elijah Chudnof (2019) based on the phenomenon of intuition identified three more types of intuition: 1. hard-won intuitions, 2. improved intuitions and 3. expertly guided intuitions.

The theoretical framework for studying intuition mainly include: 1. Dual process cognitive theory where intuition is placed in non-conscious cognitive process, 2. Process - outcome framework which analyses each type of intuition into either intuitive judgement or intuitive insight, 3 . Phenomenon based for analysing non - stereotypical intuitions.

\section{Mindfulness - definition, practice and benefits}

The term mindfulness also does not have a single precise definition. Kabat-Zinn (1994) has described mindfulness as "paying attention in a particular way: on purpose, in the present moment, and nonjudgmentally." Kristeller (1999) defined mindfulness as "bringing one's complete attention to the present experience on a moment-to-moment basis". Marlatt \& Kristeller (1999) suggested that "mindfulness is observing one's experiences with an attitude of acceptance and loving kindness". According to Segal et al. (2002) “in mindfulness practice, the focus of a person's attention is opened to admit whatever enters 
experience, while at the same time, a stance of kindly - curiosity allows the person to investigate whatever appears, without falling prey to automatic judgments or reactivity". Brown \& Ryan (2003) have stated mindfulness as "a receptive attention to and awareness of present events and experience". They also stated that mindfulness is different from behaving mechanically. Mechanical behaviour which is also described as automatic pilot occurs without awareness of one's actions. Kabat-Zinn (2003) stated that "mindfulness includes an affectionate, compassionate quality within the attending, a sense of openhearted friendly presence and interest". According to Kabat- Zinn (2003), Mindfulness is not only of experiences of the present moment prevailing in the environment but also primarily concerned about clear awareness of one's thoughts, emotions, sensations and actions. Marlatt and Bishop et al. (2004), defined mindfulness as "a process of regulating attention in order to bring a quality of non-elaborative awareness to current experience and a quality of relating to one's experience within an orientation of curiosity, experiential openness, and acceptance".

Kirk Warren Brown, Richard M. Ryan and J. David Creswell (2007) have comprehensively reviewed theoretical foundations for the effects of mindfulness. In addition to directing the attention towards the emotional sphere of an individual, mindfulness also has the potential to recognize the relationship of mutual dependence and reciprocal influence between the mind and the body. Mindfulness has the capacity to harmonize and normalize the attitude towards intrapersonal and interpersonal variables.

Meditation is one of the methods of practicing mindfulness (Kabat-Zinn, 2005). Though mindfulness is rooted in Buddhism, most of the religions advocate some type of meditation. Practicing mindful meditation technique facilitates shifting the thoughts away from usual preoccupations toward a bigger goal of life.

Several mindfulness-based intervention models have been developed for application in clinical set up, management training, problem-solving and decision-making, and for promoting general well-being of the population which include: "1, Acceptance and Commitment Therapy (ACT; Hayes, Strosahl, \& Wilson, 1999), 2. Dialectical Behavior Therapy (DBT; Linehan, 1993), 3. Mindfulness-Based Cognitive Therapy (MBCT; Segal, Williams, \& Teasdale, 2002), and 4. Mindfulness-Based Stress Reduction (MBSR; Kabat-Zinn, 1982, 1990)".

Germer, Siegel, \& Fulton (2005) has developed a variation of mindfulness - based intervention model integrating mindfulness training into individual psychotherapy from diverse perspectives.

\section{Intuition and problem-solving}

The role of intuition in problem-solving in various domains includes education, management, clinical settings etc has been empirically investigated.

Chih-Hsien Huang (2015) analysed the students' ability to decide on the truth value of mathematical statements with unknown truth values with the theoretical framework offered by Fischbein. This framework classified intuition into affirmatory intuition, conjectural intuition, anticipatory intuition, or conclusive. Analysis of the results revealed that students use affirmatory and anticipatory intuitions in deciding on truth values of mathematical statements.

A study by Chih-Hsien Huang (2015) on problem-solving styles revealed that female students used more intuitive problem-solving styles than male students.

Kenneth J.Gilhooly (2016) reviewed the beneficial impact of incubation in creative problem-solving tasks. The beneficial effect of incubation period is attributed to the theories of unconscious work (UTT) and beneficial forgetting.

Arwanto, I Ketut Budayasa and Mega Teguh Budiarto (2018) in an explorative qualitative study on students' intuition on Polya mathematical problem-solving process found that subject of student intuition in solving mathematical problems at the stage of understanding mathematical problems uses three intuition that can help understand the mathematical problems. They are: 1. Self- evident intuition 2. Intrinsic certainty intuition, and 3. Perseverance induced intuitions at the stages of Polya problem solving process.

Melvin Princea, and Constantinos Priporas (2018) have investigated the role of intuition in bridging the gap between class room learning and the required competencies for employment and career advancement in business organizations by implementing an intervention model Intuitive Awakening Elicitation (IAE). The goal of the model was on developing and nurturing students' intuitive skills for marketing decision-making. The study emphasized the need of integrating strategies to access and employ intuitive resources in academic programs. 
Elijah Chudnoff (2019) analysed the role of intuition in problem-solving ability particularly with regard to mathematical problem-solving. Contrary to the stereotypical classification of intuition identified involvement of three types of intuition based on the phenomenon associates with the process. The three types are: "hard-won intuitions, 2. improved intuitions, and 3. expertly guided intuitions".

The limited number of empirical studies on role of intuition on problem-solving / decision-making in various domains of education reveals the scope for further investigation for developing and application of models for accessing and using intuition in problem-solving and decision - making situations.

\section{Relationship between intuition, mindfulness and problem-solving / decision-making}

From the definitions, features and functions, it is obvious that the two constructs - intuition and mindfulness - fit into different domains of cognitive functions. While intuition fits into non-conscious system (system 1) of cognitive process, mindfulness is associated with awareness and therefore fits into system 2 (conscious) of cognitive domain. Therefore, these two appear to be contradictory.

Brown and Ryan (2003) have found a positive relationship between mindfulness and the extent of harmony between the explicit and implicit emotions experienced by individuals suggesting mindfulness may trigger access to non-conscious phenomenon like intuition. The term mindful intuition has entered the academic and research domains recently. Literature on studies relating to mindfulness, intuition and problem-solving / decision-making is limited in number. Analysis of available literature revealed mixed outcome regarding impact of mindfulness to trigger intuitive problem-solving.

Erik Dane Jesse H. Jones and Michael G. Pratt (2009) argue that mindfulness not only refers to awareness and attention to the present moment and events surrounding the individual, but also applies to the cognitive and emotional processes that occur within the individual.

Munich University of Applied Sciences and Ludwig-Maximilians-University Munich, has introduced the innovative Munich Model in the curriculum since 2010. The course facilitates students to learn the theory and practice of mindfulness and meditation through for-credit (ECTS) courses. The program also teaches students to incorporate these practices in their own professional work as psychologists, teachers and social workers. Research has revealed that the regular practice of meditation in addition to improving the abilities like concentration, compassion, stress management and the regulation of emotions, also enhance overall physical and mental well-being in general. The skills learnt through this course impact both performance and creativity among the students.

Robert Drummond McNaughton (2013) studied the management styles of executive meditators. The study explored the relationship between use of meditation and intuition in decision-making among executive meditators. The study found that the meditators used their intuition in the majority of their decisions. They reported their intuition was more accurate, subsequent to meditation.

In an empirical study, Carina Remmersa, Sascha Topolinskib and Johannes Michalaka (2014) found a negative relationship between trait mindfulness and performance involving intuitive judgement task.

Claire M. Zedileus and Jonathan W. Schooler (2015) have found a negative relationship between mindfulness and insight in creative problem-solving task and found that participants in more mindful state adopted analytic approach (system 2 ) to the task.

Tejpavan Singh Gandhok (2015) in his master's thesis has investigated the influence of mindfulness to the beneficial use of intuitive resources in decision-making.

Cherie elaine oertel (2016) for enhancing awareness of intuitive resources and enabling the integration of System 1 (intuition) and System 2 (rationality) cognitive processes in career decision-making developed a mindfulness-based intervention model named Post-Engagement Reflection (PER). The model was used to study post occupational engagement activity. The results of the experimental study revealed that individuals who completed the PER in along with the interview stated more intuition-related information in response to their open-ended questions than the control group.

However, the beneficial effect of mindfulness in multiple domains of cognitive function including intuition is being recognised and incorporated in several academic and executive training programs.

Melvin Princea and Constantinos Priporas (2018) have developed Intuitive Awakening Elicitation (IAE) model to bridge the gap in the required work place competencies and the knowledge and skills acquired from academic program. The study emphasized that business schools might recognize the importance of 
intuition for a new business model, in addition to their focus on traditional analytical models to effectively handle the changing situation in the business arena.

\section{Conclusion}

The analysis of the constructs intuition and mindfulness revealed that though these constructs appear to be conflicting with regard to the domain of their cognitive function, mindfulness practice could trigger intuition and contribute to solve problems / take decisions effectively. The analysis offers the scope for developing models for integrating the strategies of practicing mindfulness, and accessing and using intuition for problemsolving / decision-making, and empirical studies on their impact and mechanism of action.

\section{References}

1. Allen Newell and Herbert A. Simon, Human Problem Solving (Englewood Cliffs, NJ: Prentice Hall, 1972).

2. Andreas de Bruin The Munich Model Brings Mindfulness and Meditation to University Students International conference Future of Education.

3. Arwanto, I Ketut Budayasa, Mega Teguh Budiarto (Advances in Social Science, Education and Humanities Research (ASSEHR), volume 227, 1st International Conference on Advanced Multidisciplinary Research (ICAMR 2018).

4. Bargh, J. A. (1989). Conditional automaticity: Varieties of automatic influence in social perception and cognition. In J. S. Uleman \& J. A. Bargh (Eds.), Unintended thought (pp. 3-51). New York: Guilford Press.

5. Betsch, C. (2004). Praeferenz fuer Intuition und Deliberation. Inventar zur Erfassung von Affekt- und kognitionsbasiertem Entscheiden [Preference for Intuition and Deliberation (PID): An inventory for assessing affect- and cognition-based decision making]. Zeitschrift fuer Differentielle und Diagnostische Psychologie, 25, 179-197.

6. Bishop, S. R., Lau, M., Shapiro, S., Carlson, L., Anderson, N. C., Carmody, J., et al. (2004). Mindfulness: A proposed operational definition. Clinical Psychology: Science and Practice, 11, $230-241$.

7. Brown, K. W. \& Ryan, R. M. (2003). The benefits of being present: Mindfulness and its role in psychological well-being. Journal of Personality and Social Psychology, 84, 822-848.

8. Carina Remmersa (2015) Sascha Topolinskib and Johannes Michalaka; The Journal of Positive Psychology, 2015 Vol. No. 3, 282-292, http://dx.doi.org/10.1080/17439760.2014.950179).

9. Charles H. Kepner and Benjamin B. Tregoe, The New Rational Manager viii (Princeton: Princeton, 1981).

10. Cherie elaine oertel (2016) Dissertation submitted to the graduate degree program in Counseling Psychology and the Graduate Faculty of the University of Kansas in partial fulfillment of the requirements for the degree of Doctor of Philosophy.

11.Chih-Hsien Huang (American Journal of Educational Research, 2015, Vol. 3, No. 12, 1484-1488).

12. Claire M. Zedileus and Jonathan W. Schooler (Frontiers in psychology) 2015 Mindfull intuition Does mindfulness influence the access to intuitive processes doi: 10.3389/fpsyg.2015.00834.

13. Elijah Chudnof. Vol 98 issue 32019465 - 480; Australasian Journal of Philosophy. https://doi.org/10.1080/00048402.2019.1658121.

14. Epstein, S. (1994). Integration of the cognitive and the psychodynamic unconscious. American Psychologist, 49, 709-724.

15. Epstein, S. (2000). The rationality debate from the perspective of cognitive-experiential self theory. Behavioral and Brain Sciences, 23, 671.

16. Erik Dane Jesse H. Jones; International Review of Industrial and Organizational Psychology, 2009, Volume 24. Edited by G. P. Hodgkinson and J. K. Ford. Copyright C 2009 John Wiley \& Sons, Ltd. 
17. Gerard P. Hodgkinson1, Janice Langan-Fox2 and Eugene Sadler-Smith; British Journal of Psychology (2008), 99, 1-27.

18. Gerald P. Lopez, Lay Lawyering 32 UCLA Law Review , 2 (1984).

19. Germer, C. K., Siegel, R. D., \& Fulton, P. R. (2005). Mindfulness and psychotherapy. NY: Guilford Press.

20. Hayes, S. C. \& Strosahl, K. D. (2004). A practical guide to Acceptance and Commitment Therapy. New York: Springer.

21. Jean e. pretz, Jeffrey b. brookings , Lauren A. Carlson, Tamera Keiter Humbert, Michael roy, Meghan jones and Daniel Memmert. Journal of Behavioral Decision Making, J. Behav. Dec. Making, 27: 454-467 (2014) Published online 16 May 2014 in Wiley Online Library (wileyonlinelibrary.com) DOI: $10.1002 / \mathrm{bdm} .1820$

22. Julie Gore and Eugene Sadler-Smith Review of General Psychology · December 2011.

23. Kabat-Zinn, J. (1982). An outpatient program in behavioral medicine for chronic pain patients based on the practice of mindfulness meditation: Theoretical considerations and preliminary results. General Hospital Psychiatry, 4, 33-47.

24. Kabat-Zinn, J. (1990). Full catastrophe living: Using the wisdom of your mind and body to face stress, pain, and illness. New York: Delacorte.

25. Kabat-Zinn, J. (1994). Wherever you go, there you are: Mindfulness meditation in everyday life. New York: Hyperion.

26. Kabat-Zinn, J. (2003). Mindfulness-based interventions in context: Past, present and future. Clinical Psychology: Science and Practice, 10, 144-156.

27. Kenneth J. Gilhooly1, Incubation and Intuition in Creative Problem Solving; Frontiers in psychology, 2016, DOI - 10.3389/fpsyg_2016.01076.

28. Kirk Warren Brown, Richard M. Ryan and J. David Creswell (2007) Mindfulness: Theoretical Foundations and Evidence for its Salutary Effects Psychological Inquiry Copyright C 2007 by 2007, Vol. 18, No. 4, 211-237.

29. Klein, S. B., \& Kihlstrom, J. F. (1998). On bridging the gap between social personality psychology and neuro-psychology. Personality and Social Psychology Review, 2, 228-242.

30. Lieberman, M. D., Jarcho, J. M., \& Satpute, A. B. (2004). Evidence-based and intuition-based self knowledge: An fMRI study. Journal of Personality and Social Psychology, 87, 421-435.

31. Linehan, M. M., (1993). Cognitive-behavioral treatment of borderline personality disorder. New York: Guilford.

32. Marlatt, G. A., \& Kristeller, J. L. (1999). Mindfulness and meditation. In W. R. Miller (Ed.), Integrating spirituality into treatment (pp. 67-84). Washington, DC: APA.

33. Melvin Princea,* , Constantinos Priporas / Australasian Marketing Journal 26 (2018) 157-162 https://doi.org/10.1016/j.ausmj.2018.05.001 1441-3582/C 2018.

34. Ochsner, K. N., \& Lieberman, M. D. (2001). The emergence of social cognitive neuroscience. American Psychologist, 56, 717-734.

35. Pretz, J. E., and Totz, K. S. (2007). Measuring individual differences in affective, heuristic, and holistic intuition. Personality and Individual Differences, 43, 1247-1257. DOI: 10.1016/j.paid. 2007.03.015).

36. Robert Drummond McNaughton, The use of meditation and intuition in decision-making: reports from executive meditators A dissertation. 
37. Schroyens, W., Schaeken, W., \& Handley, S. (2003). In search of counter-examples: Deductive rationality in human reasoning. Quarterly Journal of Experimental Psychology, 56, 1129-1145

38. Segal, Z. V., Williams, J. M. G., \& Teasdale, J. D. (2002). Mindfulness-based cognitive therapy for depression: A new approach to preventing relapse. New York: Guilford.

39. Stanovich, K. E. (1999). Who is rational? Studies of individual differences in reasoning. Hillsdale, NJ: Erlbaum

40. Stanovich, K. E., \& West, R. F. (2000). Individual differences in reasoning: Implications for the rationality debate? Behavioral and Brain Sciences, 23, 645-726.

41. Tejpavan Singh Gandhok Singapore Management University Institutional Knowledge at Singapore Management University Dissertations and Theses Collection (Open Access) Dissertations and Theses.

42.Viktor Dörfler and Fran Ackermann; Management Learning (2012) 43(5) 545-564; DOI: 10.1177/1350507611434686. 